\title{
EPÍlOGO PARA DESPUÉS DE UN PASEO CON DON MARCELINO PASCUA
}

\author{
Fernando G. Benavides
}

Universital Pompeu Fabra. Barcelona.

Como cada año, desde hace ya siete, hemos vuelto a encontrarnos convocados por la figura de Marcelino Pascua. Pero en esta ocasión, además de para debatir sobre las motivaciones, actores y acciones del Nacimiento de la Sanidad Contemporánea Española (19251944), será también para despedirnos de los Encuentros coincidiendo con el Centenario de su nacimiento. Y como en todas las despedidas en ellas confluyen sensaciones diversas $y$, de alguna manera, contrapuestas. Existe, por un lado, una sensación placentera por lo que supone haber culminado una tarea. Pero, al mismo tiempo, se experimenta un sentimiento de pérdida, pues hay que decir adiós, después de una vivencia tan enriquecedora. Podemos describirlo, con palabras de Montserrat Roig, como una hora violeta.

En este balance de la experiencia, podemos decir que los Encuentros han sido como un viaje o, mejor, como un paseo, que es como define el Diccionario de la Real Academia el viaje que se hace a pie, de manera tranquila $y$ sin prisa. Un agradable paseo en la compañía de Don Marcelino nos dice bastante bien lo que estos Encuentros han significado para algunos de nosotros. Un paseo durante el cual hemos visitado ideas y hechos, nuevos y viejos, que han ido y van configurando la práctica de la Salud Pública. Pero también, éste ha

Correspondencia:

Fernando García Benavides

Universitat Pompeu Fabra

Cl Doctor Aiguader, 80.

08003 sido un paseo por las Españas: Madrid, Valencia, Barcelona, Granada, Valladolid y de nuevo, para la despedida, hemos vuelto al punto de partida.

El paseo se inició en 1991, con una visita a las estadísticas demográfico-sanitarias ${ }^{1}$. Allí nos remontamos a 1880 , cuando aparece el primer Boletín mensual de Estadísticas demográfico-sanitarias. La razón de este arranque nos pareció obvia. Estos datos, con todas sus limitaciones (sobre todo vistos desde la perspectiva actual) representaron la base, el material que dió, y sigue dando, soporte -físico- a la práctica de la Salud Pública. Es más, el desarrollo de una buena Salud Pública depende mucho de la disponibilidad de estadísticas sanitarias o de sistemas de información sanitaria, que diríamos ahora (también depende de otras cosas, como la existencia de Escuelas de Salud Pública, pero este es otro asunto). Y entre ellas, como no podía ser menos, al ir como íbamos en compañía de Marcelino Pascua, le dedicamos especial atención a las estadísticas de mortalidad y al Movimiento Natural de Población. Estos primeros pasos suponían, como señaló E. Rodríguez Ocaña, «los primeros balbuceos de las Administraciones Públicas, en materia sanitaria, en su maduración como Estado» ${ }^{2}$.

En el II Encuentro continuamos visitando y poniéndonos al día sobre aquellos primeros momentos de la Salud Pública de finales de] XIX y principios del XX, durante los años de impulso modernizador de la vida española, en los que el desarrollo de la Salud Pública era 
una de las asignaturas pendientes para «superar el secular atraso español $\aleph^{3}$. Y algunos nos enteramos de que es en 1910 cuando hace su aparición por primera vez la epidemiología como espacio profesional; a propósito de la reforma del Instituto Alfonso XIII en la que se crea la sección de Epidemiología, aunque desligada de la información sanitaria. Más como «bomberos» para apagar focos epidémicos de enfermedades contagiosas que como la inteligencia del sistema, como vino a decirnos Ferran Martínez Navarro ${ }^{4}$. Hubo que esperar a 1935 para que, bajo la influencia de Marcelino Pascua, se produjera el encuentro en España entre la epidemiología y la estadística: la Sección de Epidemiología y Desinfección pasó a denominarse Epidemiología y Estadística. Más difícil fue el encuentro entre la Salud Pública y la Universidad, dado lo alejada que estaba la Universidad de la práctica de la Salud Pública, como nos explicó $\mathrm{M}^{\mathrm{a}}$ José Báguena ${ }^{5}$. Debido a esta incapacidad crónica de la Universidad por entender y atender las necesidadęs de formación de los sanitarios, que comenzaba a demandar la administración, surge al margen de ella la Escuela Nacional de Sanidad en 1924, a pesar de que, como señaló Josep Bernabeu, desde el primer momento Pittaluga tratara de asociar la Escuela con la Universidad $^{6}$.

Con estos mimbres, sabiendo que Marcelino Pascua había sido, si no el único, sí el principal introductor de los métodos cuantitativos en el estudio de los problemas de salud a través de sus cursos en la Escuela Nacional de Sanidad y de su trabajo en el Servicio de Estadística Sanitaria de la Dirección General de Sanidad, tomamos el camino de Valencia y, dando un salto por encima de la postguerra, la autarquía y el desarrollismo, nos reunimos en el Institut Valencia d'Estudis en Salut Pública para debatir sobre un problema más reciente: los Apellidos de la Epidemiología ${ }^{7}$. Representantes de las tres familias: la epidemiología social, la clínica y la molecular explicaron sus argumentos. Más allá de los intereses de cada grupo, legítimos por supuesto, la conclusión, provisional como todas, fue que el supuesto conflicto no era para tanto y que lo que los separa no es tanto las bases epistemológicas de su teoría — al menos de momento- sino la ausencia demasiado frecuente de una necesaria síntesis que supere las limitaciones propias del empirismo, a veces demasiado ingenuo, de algunos epidemiólogos. Lo social, lo clínico y lo molecular no son más que diferentes aproximaciones posibles a un mismo problema que va desde lo molecular a lo social pasando por lo clínico. Todo ello nos llevó a pensar que posiblemente había habido un error al plantear el problema y que en nuestro afán por polemizar habíamos asignado a la epidemiología un lugar que no le corresponde. La epidemiología es el apellido, común a todos los nombres: Sociología, Clínica y Biología. El título para un próximo debate podría ser «Los nombres de la Epidemiología».

En la siguiente etapa, el Encuentro fue en Barcelona, constituyendo el primer acto del recién inaugurado Institut Universitari de Salut Pública de Catalunya. En esta parada tuvimos la oportunidad de tratar un tema que nos anticipa al futuro. Hablamos de los problemas éti$\cos$ en la investigación epidemiológica. Entre los cuales merecen mencionarse los derechos de los sujetos que participan en los estudios, la protección de los datos que proporcionan estos sujetos o los problemas éticos relacionados con la publicación de los mismos. Problemas que posiblemente en la época de Marcelino Pascua todavía no estaban planteados con la urgencia que el asunto tiene en la actualidad. De alguna manera, son los problemas propios del desarrollo. Una vez resueltos los problemas de supervivencia: disponibilidad de datos, consolidación de grupos de sanitarios en las Administraciones, incluida la Universidad etc. surgen estos nuevos problemas. Para no perdernos mucho nos hicimos acompañar durante la visita por especialistas en Ética, como Adela Cortina, y en Derecho, como José María Mena, quienes destacaron la importancia de la existencia de códigos de buena práctica consensuados por los propios investigadores y de Comités de Etica en las instituciones donde se llevan a cabo las investigaciones.

El V Encuentro fue también en otra escuela de Salud Pública, la Escuela Andaluza de Salud Pública en Granada. La más veterana de las nuevas Escuelas creadas en esta etapa. El tema elegido trató de profundizar en la rela- 
ción compleja, pero imprescindible, entre la epidemiología y la estadística ${ }^{8}$. Como tantas veces hemos oído, la aplicación de los métodos cuantitativos al análisis de los problemas de salud había constituido, desde John Graunt, una de las bases más sólidas para el desarrollo de la Salud Pública. Pero la madurez que ha ido alcanzando la estadística y la epidemiología desde los años 50 -recordando que Marcelino Pascua fue de nuevo una de las personas clave en su difusión en España- hacía necesario revisar estas relaciones. $Y$ como fue a decirnos Adela Sanz, el momento más adecuado para este emparejamiento es cuando se está diseñando el estudio, después puede ser demasiado tarde para tratar de corregir los errores de diseño epidemiológico con el análisis estadístico ${ }^{9}$. La deficiente aplicación de la estadística que se observa todavía con demasiada frecuencia en la literatura científica, como pusieron de manifiesto Segú ${ }^{10}$ y Aibar $^{11}$, es en parte una consecuencia por no tener en cuenta este principio.

Pero si la aplicación de los métodos cuantitativos ha sido uno de los factores que explican el desarrollo de la investigación en Salud Pública, no menos cierto es que la biología molecular está suponiendo también un importante estímulo para este mismo desarrollo. La relación entre la biología y la epidemiología fue el tema al que se dedicó el VI Encuentro que celebramos en Valladolid ${ }^{12}$, la ciudad natal de Don Marcelino. Después de los debates entre contagionistas y miasmáticos, entre los que destaca el que mantuvieron Snow y Farr a propósito de la famosa epidemia de cólera en Londres, con el resultado que todos conocemos, ahora parecía que el debate se reproducía de nuevo en este final de siglo entre ambientalistas y genéticos. El gen sustituye al germen, venían a decir los ambientalistas más pesimistas. Sin embargo, parece que ni siquiera la mutación, que sería lo más correcto para comparar con el germen en esta analogía, debería ser tratada de esta manera tan maniqueísta de «buenos y malos». Pues, como señaló Jaume Bertranpetit, la supervivencia del hombre sobre la tierra depende en gran medida de la capacidad de mutar de los genes en el proceso continuo de adaptación ${ }^{13}$. Es un debate recién iniciado y donde nos queda mucho que aprender.
Respecto al VII Encuentro que se publica con este epílogo poco se puede comentar. Quizá como corolario admitir que muchos de los problemas con los que se encontró Marcelino Pascua en su tiempo están aún presentes: masa crítica débil, información sanitaria limitada y, para cerrar el círculo, escaso uso de la información epidemiológica para tomar decisiones. Habrá que seguir intentando resolverlos con lo mejor de nuestra voluntad e inteligencia.

Para finalizar, me gustaría señalar que a lo largo de este recorrido han participado numerosos colegas de distintas procedencias: institucional, geográfica e intelectual. Al final han sido casi 40 los ponentes que han intervenido en alguno de los Encuentros. Al final han sido casi cuarenta los ponentes que han intervenido en algunos de los encuentros: Universidades, Escuelas de Salud Pública se reparten el protagonismo con diferentes centros de la Administración como el Centro Nacional de Epidemiología, el Ministerio de Sanidad y Consumo y el Instituto Nacional de Estadística. Es como si finalmente, después de más de 100 años, la Salud Pública empezara a echar raíces en la Universidad y en la Administración. Pero en este capítulo de los amigos encontrados, también hemos de registrar pérdidas. Enrique Nájera nos dejó en 1994. Enrique fue una de las personas que mantuvo vivo durante la larga dictadura lo mejor de la tradición sanitaria republicana que representa Marcelino Pascua y la supo transmitir a la generación de sanitarios que surge durante el período de la transición política (1975-1982). A muchos de nosotros.

Ha sido este, pues, un viaje por el pasado y también por el presente. Pero sobre todo, ha sido un viaje pensando en el futuro. Sin apenas darnos cuenta hemos creado, inventado diría Hobsbawn, una tradición. Y esto significa que tenemos voluntad de perpetuarnos, que queremos tener una memoria colectiva que nos obligue - voluntariamente - a todos, a nuestros antecesores, a nosotros y a nuestros sucesores a «hacer el presente siempre presente». Tradición significa, del latín traditio, transmisión de bienes, materiales y espirituales. Tener en cuenta el pasado, mezclarlo con el presente y transmitirlo al futuro es todo el afán que han albergado los Encuentros. Esperamos que ha- 
yan contribuido a la recuperación de la memoria histórica y de una identidad para la Salud Pública española del futuro.

Otros muchos asuntos han quedado pendientes, pues, aunque siete años no es un periodo corto, los temas, curiosidades y preocupaciones son siempre numerosas. Uno de estos temas que ha quedado pendiente es el de la relación entre la Salud Pública y las Ciencias Sociales, como la economía, la sociología, la política, la demografía, etc. De otros modos, por otras personas, ahora esperamos que la tradición continúe. Quizás en forma de Conferencia anual coincidiendo con las Reuniones Científicas de la SEE. Sea como fuere, creo que podemos felicitarnos todos por haber llegado hasta aquí, agradeciendo además esta tarde tan agradable, pasada con todos vosotros.

\section{BIBLIOGRAFÍA}

1. I Encuentro Marcelino Pascua: Estadísticas Demográfico-sanitarias (14 de junio de 1991). Madrid: Centro Nacional de Epidemiología. Instituto de Salud Carlos III. Ministerio de Sanidad y Consumo; 1992.

2. Rodríguez Ocaña E. La estadística en la Administración Sanitaria española del siglo veinte. En: I Encuentro Marcelino Pascua: Estadísticas Demográfico-sanitarias (14 de junio de 1991). Madrid: Centro Nacional de Epidemiología. Instituto de Salud Carlos III. Ministerio de Sanidad y Consumo; 1992.p. 47-72.

3. II Encuentro Marcelino Pascua (12 de junio de 1992). La epidemiología en el desarrollo de la Salud Pública española. Rev San Hig Pública 1994; 68 (monográfico).
4. Martínez Navarro F. Salud Pública y desarrollo de la epidemiología en la España del siglo XX. Rev San Hig Pública 1992; 68: 29-43.

5. Baguena MJ. La higiene y la Salud Pública en el marco universitario español. Rev San Hig Pública 1992; 68: 91-96.

6. Bernabeu J. El papel de la escuela Nacional de Sanidad en el desarrollo de la salud Pública en España, 1924-1934. Rev San Hig Pública 1992; 68: 65-89.

7. III Encuentro Marcelino Pascua (11 de junio de 1993). La epidemiología y sus apellidos. Barcelona: Institut Universitari de salut Pública de Catalunya; 1995.

8. V Encuentro Marcelino Pascua (16 de junio de 1997). La epidemiología y la estadística. Granada: Escuela Andaluza de Salud Pública; 1996.

9. Sanz MA. La epidemiología y la estadística. En: V Encuentro Marcelino Pascua (16 de junio de 1997). La epidemiología y la estadística. Granada: Escuela Andaluza de Salud Pública; 1996.p.35-44.

10. Segú JL. Abusos de la estadística en medicina. En: V Encuentro Marcelino Pascua (16 de junio de 1997). La epidemiología y la estadística. Granada: Escuela Andaluza de Salud Pública; 1996.p. 63-73.

11. Aibar C. Uso de la estadística en las publicaciones médicas. En: V Encuentro Marcelino Pascua (16 de junio de 1997). La epidemiología y la estadística. Granada: Escuela Andaluza de Salud Pública; 1996.p. 45-61.

12. VI Encuentro Marcelino Pascua ( 14 de junio de 1996). Epidemiología y Biología (en prensa).

13. Bertranpetit J. Una historia natural de la enfermedad genética. En: VI Encuentro Marcelino Pascua (14 de junio de 1996). Epidemiología y Biología (mimeo). 